\title{
Les états de conscience altérée : études comportementales et de neuroimagerie
}

\author{
Disorders of consciousness: behavioral and
}

\author{
neuroimaging studies
}

\section{Titre abrégé : Troubles de la conscience : clinique et neuroimagerie}

\author{
Leandro R. D. Sanz ${ }^{1}$, Steven Laureys ${ }^{1}$, Olivia Gosseries ${ }^{1}$ \\ 1. \\ Coma Science Group, GIGA Consciousness, University and University Hospital of Liège, Liège, Belgium \\ Auteur correspondant : Leandro R. D. Sanz, leandro.sanz@uliege.be, +3243663915
}

\section{Résumé en français}

Les troubles de l'état de conscience après un coma représentent à la fois un défi clinique pour les équipes soignantes et un sujet d'intérêt pour la recherche sur la conscience humaine. Afin d'uniformiser la nomenclature dans ce domaine en constante évolution, une classification définit à présent de manière claire les différents stades de conscience altérée et leurs signes cliniques diagnostiques. L'évaluation comportementale de ces patients repose aujourd'hui sur des échelles standardisées qui doivent être réalisées de manière répétée. Toutefois, le seul examen clinique ne suffit pas car des troubles sensoriels, moteurs, phasiques ou de la vigilance peuvent masquer la présence d'une perception consciente. De nouvelles méthodes de neuroimagerie et de neurophysiologie utilisant des outils d'analyse novateurs permettent de préciser le diagnostic clinique et d'évaluer ces patients de manière plus précise. Sur le plan thérapeutique, la neurostimulation non- 
invasive ainsi que certains agents pharmacologiques ont démontré des effets positifs sur la récupération, mais des études contrôlées par placebo à plus grande échelle sont nécessaires pour déterminer plus précisément l'efficacité et le taux de réponse à certains traitements. Une intégration multimodale de ces nouvelles techniques diagnostiques et thérapeutiques est recommandée afin de proposer aux patients une prise en charge personnalisée.

\section{Résumé en anglais}

Disorders of consciousness after a coma are both a clinical challenge for caregivers and a unique research opportunity on human consciousness for neuroscientists. To set standards in this constantly evolving field, a nosological classification now clearly defines the different states of impaired consciousness and their diagnostic clinical signs. The gold-standard behavioral evaluation of these patients is based on standardized scales that must be administered repeatedly. However, the sole clinical examination is not self-sufficient as sensorimotor impairments, aphasia or fluctuations of vigilance levels can conceal the presence of conscious awareness. New methods using neuroimaging and neurophysiology combined with innovative computational tools can complement the clinical diagnosis and help assess these patients more accurately. Regarding treatment, non-invasive neurostimulation and pharmacological agents have demonstrated positive effects on recovery, but larger scale placebo-controlled clinical trials are needed to determine their efficacy and response rate more accurately. A multimodal integration of these new diagnostic and therapeutic techniques is necessary to offer patients with severe brain injury a more personalized and adapted care.

Mots-clés: Troubles de la conscience ; Coma; État végétatif ; Etat de conscience minimale ; Neuroimagerie ; Neurophysiologie ; Diagnostic ; Pronostic ; Traitement

Keywords: Consciousness disorders; Coma; Vegetative state; Minimally conscious state; Neuroimaging ; Neurophysiology ; Diagnosis ; Prognosis ; Treatment 


\section{Introduction}

Suite à une lésion cérébrale importante, les patients peuvent se retrouver dans différents états de conscience altérée. Leur prise en charge représente un défi diagnostique et thérapeutique pour les cliniciens. Cependant, ces pathologies fournissent aussi une opportunité unique pour les chercheurs d'étudier les processus cérébraux qui génèrent et maintiennent la conscience humaine. L'intérêt grandissant pour ce sujet d'étude ainsi que les récentes avancées technologiques au niveau des méthodes d'évaluations et d'analyse des données ont permis une progression impressionnante de la compréhension des mécanismes impliqués dans les processus conscients et leurs altérations. L'évaluation clinique de ces patients a gagné en précision grâce à l'utilisation d'échelles comportementales standardisées et elle est aujourd'hui complétée par une panoplie de techniques utilisant la neurophysiologie et la neuroimagerie afin d'augmenter les chances de détecter des signes de conscience, aidant ainsi à guider le pronostic, les choix thérapeutiques et les questions éthiques.

\section{Les troubles de la conscience}

Si aucune définition de la conscience n'est aujourd'hui unanimement acceptée par la communauté scientifique, il apparaît que deux composantes essentielles sont nécessaires pour produire une expérience consciente : le niveau d'éveil et la perception consciente [1]. L'éveil, objectivé par l'ouverture des yeux, est principalement contrôlé par la formation réticulée au sein du tronc cérébral et ses projections. La perception consciente, parfois appelée expérience subjective, correspond à la capacité d'interaction avec l'environnement et de représentation de soi, ce qui est véhiculé par l'ensemble des fonctions cognitives et affectives de l'individu et dépend essentiellement du cortex cérébral (Figure 1). 


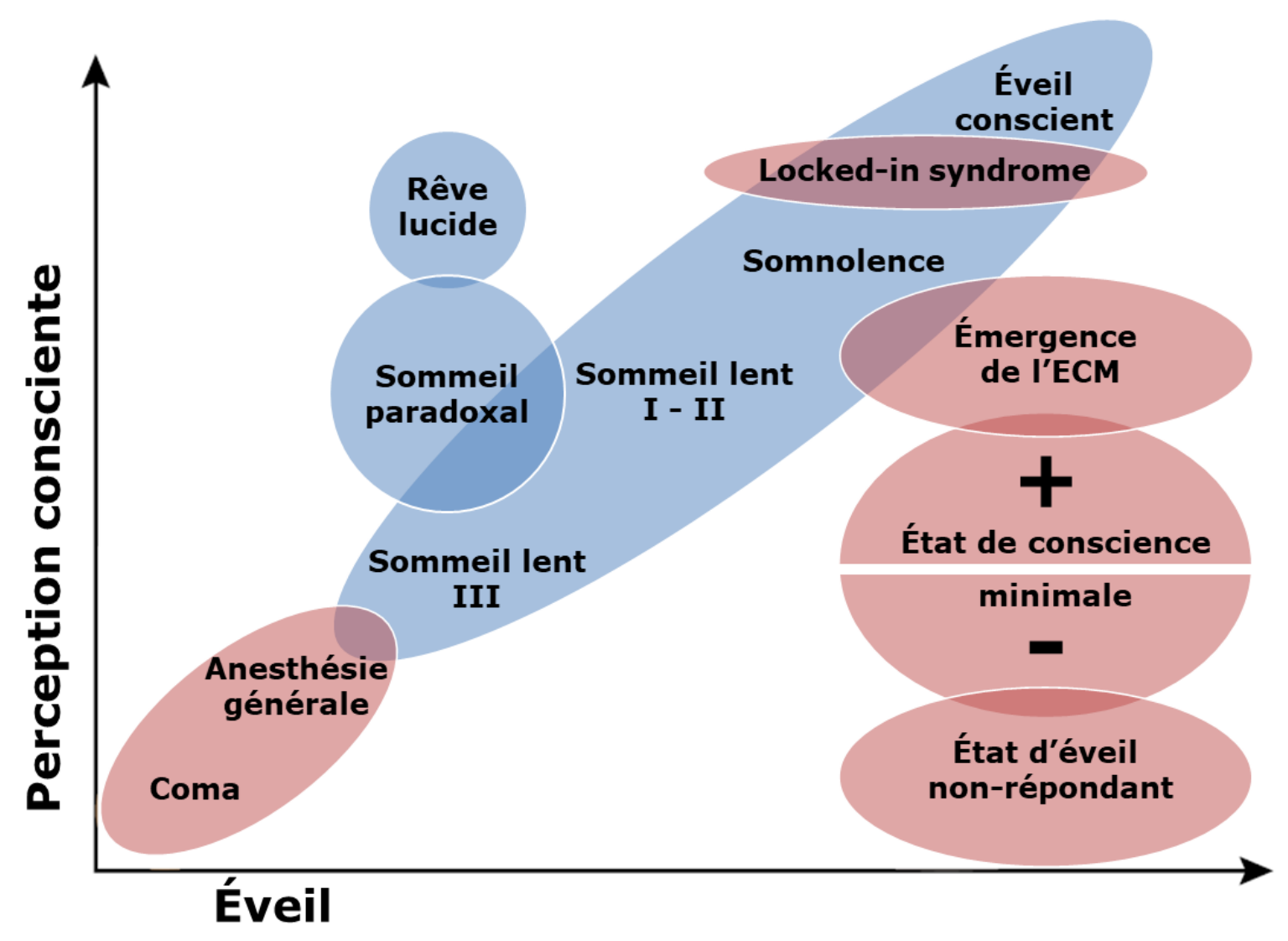

Figure 1. Les différents états physiologiques (en bleu) et pathologiques (en rouge) de conscience altérée, en fonction des deux composantes principales de la conscience : le niveau d'éveil et la perception consciente. Adapté de [1].

Au cours de l'endormissement, on observe une diminution harmonieuse des niveaux d'éveil et de perception consciente proportionnelle à la profondeur du stade de sommeil lent. Au contraire, durant le sommeil paradoxal, le niveau de perception consciente élevé lié aux rêves contraste avec le niveau d'éveil qui reste bas.

Suite à une lésion cérébrale importante, les patients présentent généralement une phase initiale de coma durant laquelle aussi bien l'éveil que la perception consciente sont absents, et leurs yeux restent fermés [2]. Ceux qui survivent à cette période aigue et transitoire ouvrent d’abord les yeux, témoignant d'une récupération du niveau d'éveil et donc de la sortie du coma, mais peuvent présenter des niveaux très variables de perception consciente. La quantification de cette perception consciente grâce à la 
recherche de signes cliniques de conscience permet aux cliniciens de stratifier ces patients en différents états de conscience altérée.

L'état d'éveil non-répondant (ENR), anciennement appelé état végétatif, est caractérisé par la présence d'éveil avec ouverture des yeux spontanée ou après stimulations (auditives, tactiles, et/ou douloureuses) mais l'absence de tout comportement volontaire reproductible [3]. Les cycles veillesommeil peuvent toutefois être préservés chez ces patients. Cet état était auparavant considéré comme permanent après une période de 3 mois pour une lésion non-traumatique et 12 mois pour une lésion traumatique, mais la mise en évidence d'une récupération possible après de plus longs intervalles en ENR a conduit à l'abandon de cette distinction [4]

L'état de conscience minimale (ECM) est défini par la présence d'éveil ainsi que de signes de conscience non-équivoques et reproductibles [5]. II est aujourd'hui sous-divisé en deux entités cliniques. L'ECM plus $(E C M+)$ se distingue par des capacités de compréhension ou de production langagière, illustrées par des réponses reproductibles à la commande, des verbalisations intelligibles ou une communication intentionnelle. L'ECM moins (ECM-) est quant à lui caractérisé par des signes de conscience plus rudimentaires, tels que la poursuite ou la fixation visuelle, la reconnaissance ou la manipulation d'objets, la localisation d'objets ou de stimuli douloureux ainsi que les réactions motrices automatiques [6]. Le niveau de perception consciente de ces patients est très souvent fluctuant en fonction du moment de la journée, du niveau de motivation et des possibles complications médicales. Pour cette raison, il est essentiel de procéder à une évaluation comportementale répétée afin d'obtenir un diagnostic fiable [7].

Lorsque les patients récupèrent la faculté de communiquer (en répondant correctement à des questions simples par un code oui/non préétabli) ou utilise des objets de manière fonctionnelle, ils sont considérés comme émergents de l'ECM. Cependant, ces patients présentent fréquemment un état confusionnel, avec des épisodes d'agitation ou des atteintes importantes de l'attention, de mémoire et d'orientation [8]. 
Il est important de mentionner également le syndrome d'enfermement, ou locked-in syndrome, souvent consécutif à une occlusion du tronc basilaire entraînant une ischémie du pont. Dans sa forme complète, cet état est caractérisé par des niveaux d'éveil et de perception consciente normaux mais une paralysie complète de tous les groupes musculaires à l'exception des muscles oculaires, permettant une communication exclusivement via les mouvements verticaux des yeux. Une évaluation non-exhaustive de ces patients peut mener à un diagnostic erroné de trouble de la conscience et une prise en charge inadaptée [9].

Si le syndrome d'enfermement est un exemple probant de la nécessité d'effectuer un diagnostic différentiel méticuleux, il est également essentiel de stratifier de manière correcte et fiable les différents états de conscience altérée. Tout d'abord, cette classification permet une évaluation plus précise du pronostic clinique à long terme. En effet, il apparaît que plus d'un tiers des patients diagnostiqués en ECM un mois après la lésion cérébrale en ont émergé après un an, alors que seulement $11 \%$ des patients en ENR ont atteint ce stade après le même intervalle [10]. Un diagnostic fiable permet également de proposer des options thérapeutiques ciblées et adaptées à chaque individu, car l'efficacité des traitements disponibles est dépendante du diagnostic des patients traités [11]. De plus, la connaissance précise de la capacité de conscience d'un patient permet de guider la conduite à tenir sur le plan éthique, notamment dans les cas de décisions de fin de vie et de retrait thérapeutique [12].

\section{L'évaluation clinique}

Pendant longtemps, l'évaluation comportementale des patients présentant des troubles de la conscience a reposé uniquement sur l'échelle de Glasgow, complétée en 2005 par le score FOUR qui ajoute notamment la poursuite visuelle et les réflexes du tronc cérébral. Rapides et aisées à utiliser dans le cadre des unités de soins intensifs, ces échelles présentent toutefois une faible sensibilité à détecter certains signes de conscience, ce qui peut amener à sous-estimer le diagnostic d'un patient [13]. L'échelle de récupération du coma (Coma Recovery Scale - Revised ; CRS-R) [14] propose une 
évaluation plus complète des capacités du patient sous la forme de six sous-échelles reflétant les fonctions auditive, visuelle, motrice, oromotrice, la communication et l'éveil (Tableau 1). Les signes de conscience les plus fréquemment observés chez les patients diagnostiqués en ECM au moyen de la CRS-R sont la réponse reproductible à la commande, la fixation et la poursuite visuelle, la localisation des stimuli nociceptifs et les réponses motrices automatiques [15]. Cette échelle a démontré une sensibilité et une spécificité élevée si elle est administrée de manière répétée (au moins cinq évaluations) [7], et de manière optimale (en utilisant un miroir pour tester la poursuite visuelle [16] et le prénom du patient pour la localisation auditive [17], par exemple). 
Tableau 1. Caractéristiques cliniques fréquemment observées pour différents états de conscience.

ENR : Etat d'éveil non-répondant ; ECM-: Etat de conscience minimale moins; ECM+: Etat de conscience minimale plus; Emergence ECM : Emergence de l'état de conscience minimale ; ${ }^{*}$ : signes cliniques diagnostiques.

\begin{tabular}{|c|c|c|c|c|c|c|}
\hline & Coma & ENR & ECM- & ECM+ & Emergence ECM & Locked-in \\
\hline $\begin{array}{l}\text { Fonction } \\
\text { auditive }\end{array}$ & Absence & $\begin{array}{l}\text { Absence / } \\
\text { Sursaut au } \\
\text { bruit / } \\
\text { Localisation } \\
\text { des sons }\end{array}$ & $\begin{array}{c}\text { Sursaut au bruit / } \\
\text { Localisation des } \\
\text { sons }\end{array}$ & $\begin{array}{l}\text { Réponse à la } \\
\text { commande } \\
\text { reproductible ou } \\
\text { systématique* }\end{array}$ & $\begin{array}{l}\text { Réponse à la } \\
\text { commande } \\
\text { reproductible ou } \\
\text { systématique }\end{array}$ & $\begin{array}{l}\text { Réponse à la } \\
\text { commande } \\
\text { systématique }\end{array}$ \\
\hline $\begin{array}{l}\text { Fonction } \\
\text { visuelle }\end{array}$ & Absence & $\begin{array}{l}\text { Absence / } \\
\text { Clignement à } \\
\text { la menace }\end{array}$ & $\begin{array}{l}\text { Fixation ou } \\
\text { poursuite visuelle* } \\
\text { / Localisation ou } \\
\text { reconnaissance } \\
\text { d'objets* }\end{array}$ & $\begin{array}{l}\text { Fixation ou } \\
\text { poursuite } \\
\text { visuelle / } \\
\text { Localisation ou } \\
\text { reconnaissance } \\
\text { d'objets } \\
\end{array}$ & $\begin{array}{l}\text { Fixation ou } \\
\text { poursuite } \\
\text { visuelle / } \\
\text { Localisation ou } \\
\text { reconnaissance } \\
\text { d'objets } \\
\end{array}$ & $\begin{array}{l}\text { Reconnaissance } \\
\text { d'objets }\end{array}$ \\
\hline $\begin{array}{l}\text { Fonction } \\
\text { motrice }\end{array}$ & \begin{tabular}{|l} 
Absence / \\
Réaction \\
anormale à la \\
douleur
\end{tabular} & \begin{tabular}{|l} 
Absence / \\
Réaction \\
anormale à la \\
douleur \\
\end{tabular} & $\begin{array}{c}\text { Réactions } \\
\text { automatiques* / } \\
\text { Manipulation } \\
\text { d'objets* } \\
\end{array}$ & $\begin{array}{c}\text { Réactions } \\
\text { automatiques / } \\
\text { Manipulation } \\
\text { d'objets } \\
\end{array}$ & $\begin{array}{l}\text { Utilisation } \\
\text { fonctionnelle } \\
\text { d'objets* }\end{array}$ & $\begin{array}{l}\text { Absence / } \\
\text { Minimale }\end{array}$ \\
\hline $\begin{array}{l}\text { Fonction } \\
\text { oromotrice }\end{array}$ & $\begin{array}{l}\text { Absence / } \\
\text { Mouvements } \\
\text { réflexes }\end{array}$ & $\begin{array}{l}\text { Absence / } \\
\text { Mouvements } \\
\text { réflexes }\end{array}$ & $\begin{array}{l}\text { Mouvements } \\
\text { réflexes / } \\
\text { Vocalisations }\end{array}$ & $\begin{array}{l}\text { Verbalisation } \\
\text { intelligible* }\end{array}$ & $\begin{array}{l}\text { Verbalisation } \\
\text { intelligible }\end{array}$ & $\begin{array}{l}\text { Absence / } \\
\text { Minimale }\end{array}$ \\
\hline Communication & Absence & Absence & Absence & $\begin{array}{l}\text { Communication } \\
\text { intentionnelle* }\end{array}$ & $\begin{array}{l}\text { Communication } \\
\text { fonctionnelle* }\end{array}$ & $\begin{array}{l}\text { Via mouvements } \\
\text { oculaires* }\end{array}$ \\
\hline Eveil & Absence & $\begin{array}{l}\text { Ouverture } \\
\text { des yeux à la } \\
\text { stimulation* }\end{array}$ & Fluctuant & Fluctuant & Fluctuant & $\begin{array}{l}\text { Préservé / } \\
\text { Fluctuant }\end{array}$ \\
\hline
\end{tabular}

Malgré l'utilisation optimale d'une échelle comportementale standardisée et complète telle que la CRS-R, il existe d'autres variables qui peuvent entraver la détection de la conscience chez ces patients. En effet, la présence d'une aphasie, de troubles moteurs (parésie, paralysie) ou sensoriels (cécité, surdité), de douleurs importantes, de médicaments sédatifs, de complications médicales, d'un manque de motivation ou d'un niveau de vigilance fluctuant sont autant de facteurs pouvant amener à une sous-évaluation du niveau de conscience. Ainsi, on estime que l'évaluation uniquement clinique des patients avec des troubles de la conscience pourrait mener à un diagnostic erroné dans un tiers des 
cas [18]. Une évaluation plus fine du niveau de douleur peut être réalisée à l'aide d'une autre échelle standardisée, la Nociception Coma Scale - Revised, permettant d'adapter l'antalgie [19]. Certains signes cliniques tels que la présence de comportements émotionnels contextualisés [5], la localisation de stimuli auditifs [17], la résistance à l'ouverture des yeux [20] ou la présence d'une déglutition efficace ont été proposés [21] comme critères diagnostiques de l'ECM, mais leur validité en tant que signes de conscience est encore débattue. Dès lors, il est crucial de disposer de méthodes diagnostiques indépendantes de la participation du patient et de ses facultés sensori-motrices, telles que la neurophysiologie et la neuroimagerie.

\section{L'évaluation par neuroimagerie et neurophysiologie}

Le récent essor des nouvelles techniques d'imagerie cérébrale, qui permettent de mesurer la structure et l'activité cérébrale en un temps record et de manière non-invasive, a ouvert de nouvelles perspectives dans l'évaluation des patients souffrant de troubles de la conscience. En particulier, ces outils sont capables de déceler des marques d’activité cérébrale indépendamment des capacités comportementales des patients.

Les images structurelles, principalement celles acquises grâce à l'imagerie par résonance magnétique (IRM), permettent d'évaluer en détail l'architecture du cerveau ainsi que de l'intégrité anatomique de ses structures. Les récentes méthodes d'analyses par morphométrie comparent le volume de matière grise dans le cerveau d'un patient et dans le cerveau de sujets sains, mettant ainsi en évidence les régions les plus touchées par une perte neuronale [22]. Parallèlement, les séquences d'imagerie par tenseur de diffusion donnent une information sur la préservation des fibres de matière blanche et donc sur la structure des connexions cérébrales. Cette modalité a très récemment démontré une capacité à prédire le pronostic neurologique à long-terme des patients après un arrêt cardiaque et pourrait ainsi constituer le premier outil prédictif pour la stratification précoce des patients cérébrolésés aux soins intensifs [23]. 
Mesurant en temps réel la fonction cérébrale, l'imagerie fonctionnelle est encore plus informative pour l'évaluation de la conscience. On peut distinguer trois grands types de paradigmes:

1) Les tâches actives requièrent la participation du sujet et donc la capacité de comprendre et intégrer les instructions. Ce type d'examens possède une bonne spécificité à détecter la conscience (un résultat positif indiquant de manière fiable la présence de conscience), mais une faible sensibilité (certains patients conscients mais aphasiques entraîneront un résultat négatif, par exemple). L'IRM fonctionnelle permet de réaliser des tâches actives en demandant au patient de se représenter en train de jouer au tennis ou de parcourir les pièces de sa maison, durant l'acquisition [24]. Ces tâches sont corrélées à des zones d'activation cérébrale bien précises chez les sujets sains, et il est possible de confirmer qu'un patient réalise la tâche mentale demandée si un motif similaire d'activation est retrouvé, témoignant de la présence d'une perception consciente. Une communication binaire a pu être établie avec un patient capable de réaliser les deux tâches mentales correctement, en s'imaginant jouer au tennis pour dire « oui » et déambuler dans sa maison pour dire « non », alors qu'aucune autre forme de communication verbale ou motrice n'était possible chez lui [25]. D'autres systèmes plus facilement utilisables au chevet des patients utilisent des tâches actives afin de mettre en place une communication avec ces patients. En interprétant les variations de signal d'un électroencéphalogramme (EEG) lors de la réalisation de tâches mentales spécifiques, des interfaces cerveau-ordinateur visent à établir une communication en temps réel [26]. D’abord développées pour les patients en locked-in syndrome, ces techniques sont désormais testées chez des patients avec des troubles de la conscience. D'autres interfaces similaires sont basées sur la spectroscopie proche infrarouge [27], les variations du diamètre pupillaire [28], le pH salivaire [29] ou le contrôle de la respiration [30], mais le succès de leur implémentation reste très variable d'un patient à l'autre.

2) Les tâches passives enregistrent l'activité cérébrale durant une stimulation sensorielle, sans participation active du sujet. Cette modalité diminue les faux négatifs dus à des troubles moteurs ou phasiques, mais les déficiences sensorielles (cécité, surdité) restent une entrave à la détection de 
conscience. De plus, certains signes enregistrés passivement n'impliquent pas nécessairement la présence d'une perception consciente, augmentant ainsi les faux négatifs. Par exemple, on peut mesurer les variations d'activité EEG en réponse à la présentation de stimuli auditifs et sonder la présence de potentiels évoqués, gages d'une fonction auditive préservée mais pas nécessairement signes de conscience [31,32].

3) Les enregistrements au repos sont aujourd'hui des outils de choix pour la détection de conscience car ils ne dépendent pas des capacités sensori-motrices des patients, ce qui leur confère une grande sensibilité à déceler des signes de conscience. La tomographie par émission de positrons est utilisée depuis plus de 20 ans pour évaluer le métabolisme cérébral au repos des patients cérébro-lésés, une mesure qui est corrélée au niveau de conscience cliniquement objectivé [18]. L'IRM fonctionnelle au repos a permis de mettre en évidence certains réseaux importants pour la génération de la conscience, dont le réseau du mode par défaut, vraisemblablement impliqué dans la conscience de soi et la pensée libre. Des altérations de la connectivité fonctionnelle ont également pu être identifiées chez des patients en ENR au moyen de l'IRM fonctionnelle [33]. En utilisant l'EEG à haute densité au repos, une méthode moins coûteuse et plus pratique d'utilisation, de nouvelles méthodes d'analyse de la connectivité dynamique basées sur la théorie des graphes ont démontré leur capacité à prédire avec précision le diagnostic clinique des patients grâce à seulement quelques minutes d'enregistrement (Figure 2) [34]. Une autre méthode utilise une panoplie de marqueurs EEG reflétant la puissance, le degré d'information et la connectivité du signal, qui sont analysés par un algorithme informatique de classification utilisant l'apprentissage automatique (machine learning), calculant ensuite une probabilité d'être conscient pour chaque patient [35]. La stimulation magnétique transcrânienne couplée à l'EEG, qui consiste à mesurer la réponse électrique cérébrale à une perturbation induite par un champ magnétique, offre également un outil diagnostique intéressant. La complexité de la réponse est estimée grâce à un index unique qui intègre la durée, l'étendue et l'aspect du signal EEG, ce qui permet également de prédire de manière fiable la capacité de conscience d'un patient [36]. 

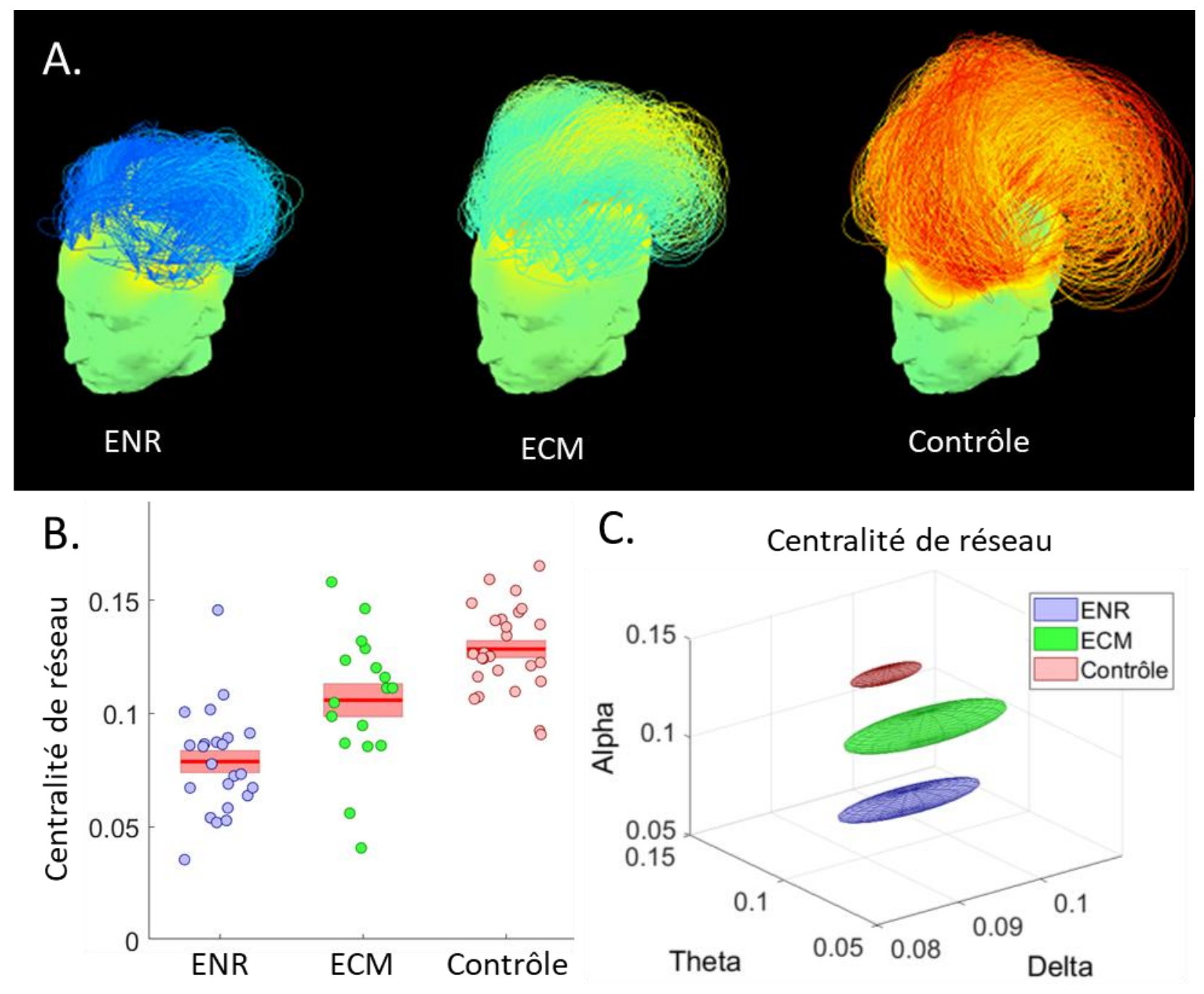

Figure 2. Analyse de la connectivité dynamique par électroencéphalographie à haute-densité de repos, pour différents groupes diagnostiques. (A) Topographes 3D en bande alpha des réseaux corticaux. Le code couleur et la hauteur des boucles sont proportionnels au degré de connectivité entre deux nœuds du réseau. (B) Mesures du degré de centralité des réseaux corticaux pour la bande alpha, un indicateur de la connectivité dynamique globale. (C) Représentation 3D du degré de centralité des réseaux corticaux pour les bandes alpha, thêta et delta. L'intégration de ces trois bandes de fréquences permet une stratification plus précise des patients. ENR : État d'éveil non-répondant ; ECM : État de conscience minimale. Images du Coma Science Group, selon les méthodes décrites dans [34].

L'utilisation de ces nouvelles méthodes diagnostiques a entraîné la création d'une nouvelle entité nosologique, l'ECM*, pour décrire les patients qui ne présentent aucun critère clinique de conscience 

ou de neurophysiologie (Figure 3).

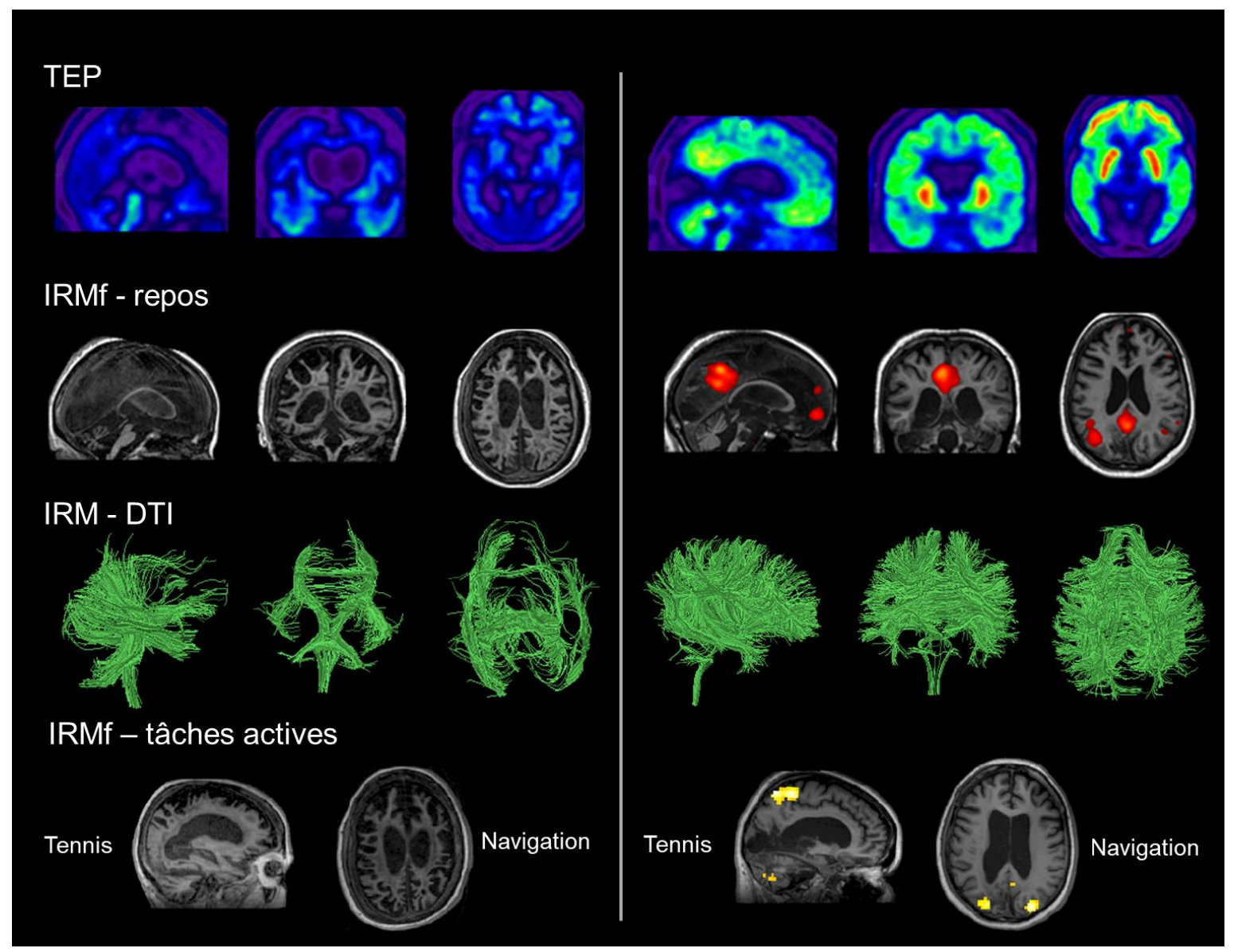

Figure 3. Différentes techniques de neuroimagerie pour l'évaluation de la conscience chez deux patientes cliniquement en état d'éveil non-répondant. Les résultats structurels et fonctionnels de la patiente de gauche sont compatibles avec son diagnostic alors que la patiente de droite montre des signes d'activité cérébrale indiquant la présence d'une perception consciente. Assemblage d'images de tomographie par émission de positrons (TEP), d'imagerie par résonance magnétique fonctionnelle au repos (IRMf - repos ; réseau du mode par défaut), d'imagerie par tenseur de diffusion (IRM - DTI) et de tâches actives exécutées dans l'IRMf (IRMf - tâches actives). De gauche à droite, vues sagittales, coronales et axiales du cerveau, pour chaque patiente. Adapté de [37] avec permission.

\section{Les options thérapeutiques}


Au vu du nombre grandissant de patients souffrant de troubles chroniques de la conscience, qui nécessitent de mobiliser des moyens financiers et logistiques énormes pour leur prise en charge quotidienne, le développement de traitements pour améliorer leur récupération est d'une importance majeure.

Parmi les méthodes pharmacologiques qui visent à accélérer ou améliorer la récupération de la conscience, deux classes d'agents se sont distingués par leurs effets positifs chez cette population de patients : les agonistes de la dopamine et les agonistes de l'acide gamma-aminobutyrique (GABA). Plusieurs agents dopaminergiques, utilisés essentiellement pour le traitement de la maladie de Parkinson, ont démontré une efficacité clinique pour la récupération au sein d'études pilotes [38]. Cependant, seule l'amantadine a prouvé une accélération de la récupération durant la prise du traitement dans un essai clinique randomisé contrôlé par placebo, sans différence significative de récupération avec le groupe contrôle 2 semaines après l'arrêt du traitement [39]. Les agents GABAergiques sont majoritairement employés comme somnifère. Le zolpidem, connu pour son action hypnotique rapide et sa sécurité d'utilisation, produit une amélioration transitoire du niveau d'éveil et des capacités cognitives chez certains patients présentant des troubles de la conscience. Cet effet paradoxal, survenant après la prise d'une seule dose et ne durant que quelques heures, ne serait présent que chez 5 à $7 \%$ de la population étudiée [40].

Les approches non-pharmacologiques se sont principalement concentrées sur la neurostimulation. Suite au succès de la stimulation cérébrale profonde pour le traitement des symptômes de la maladie de Parkinson, cette méthode a également été utilisée pour les états de conscience altérée. Des effets comportementaux anecdotiques ont pu être obtenus en ciblant le thalamus central, mais seulement 10 à $20 \%$ des patients remplissent les critères pour bénéficier de cette intervention invasive qui présente par ailleurs des risques d'effets secondaires importants [41,42]. En comparaison, la stimulation transcrânienne à courant continu est une méthode non-invasive qui permet de stimuler des régions ciblées du cortex à travers le scalp grâce à un courant électrique de faible intensité, de 
manière plus sûre et moins couteuse. Une étude contrôlée par placebo a notamment montré une amélioration transitoire des signes de conscience observables après une stimulation de 20 minutes sur le cortex préfrontal dorsolatéral, chez près de la moitié des patients en ECM mais pas chez les patients en ENR [43]. D’autres méthodes utilisant la stimulation magnétique transcranienne répétée [44], la stimulation du nerf vague [45] et les ultrasons de faible intensité [46] ont obtenu des résultats positifs sur des cas isolés.

Aucun traitement n'a encore prouvé une efficacité à long-terme pour la récupération des troubles de la conscience, et de nouvelles études sont requises pour déterminer plus précisément l'efficacité réelle de certains candidats potentiels.

\section{Conclusion}

La prise en charge des patients cérébro-lésés après un coma est un défi majeur qui implique à la fois une démarche clinique, la santé publique, l'éthique médicale et la recherche neuroscientifique. Depuis le développement des unités de soins intensifs et de la ventilation mécanique, le nombre de patients qui développent des troubles chroniques de la conscience augmente sans cesse. De nouveaux outils combinant des techniques d'imagerie cérébrale de pointe avec des algorithmes informatiques novateurs offrent aujourd'hui de nouvelles clés pour établir un diagnostic précis, en complément de I'utilisation répétée d'échelles cliniques standardisées. La découverte de signes de conscience chez des patients cliniquement non-répondants grâce à l'imagerie fonctionnelle suggère qu'il faut rester particulièrement prudents dans notre jugement clinique initial et souligne que l'absence de preuves de conscience n'équivaut pas à une preuve de son absence. Cette prise en charge multimodale permet de mieux stratifier cette population de patients et ainsi estimer le pronostic de récupération à longterme, proposer des traitements adaptés et aider à la prise de décisions de fin de vie. Il est important de souligner que les possibilités thérapeutiques demeurent très limitées; la recherche future doit s'atteler au développement de traitements efficaces, sûrs et applicables au lit du patient.

\section{Références}


1 Laureys S. The neural correlate of (un)awareness: Lessons from the vegetative state. Trends Cogn Sci $2005 ; 9$ : 556-559.

2 Posner JB, Saper CB, Schiff N, et al. Plum and Posner's Diagnosis of Stupor and Coma. Oxford University Press; 2008.

3 Rutgeerts P, Robert L, Helmut M, et al. Medical Aspects of the Persistent Vegetative State. N Engl J Med $1994 ; 330$ : 1499-1508.

4 Laureys S, Celesia GG, Cohadon F, et al. Unresponsive wakefulness syndrome: A new name for the vegetative state or apallic syndrome. BMC Med $2010 ; 8: 2-5$.

5 Giacino JT, Ashwal S, Childs N, et al. The minimally conscious state. Neurology $2002 ; 58: 349-$ 353.

6 Bruno M-A, Vanhaudenhuyse A, Thibaut A, et al. From unresponsive wakefulness to minimally conscious PLUS and functional locked-in syndromes: Recent advances in our understanding of disorders of consciousness. J Neurol $2011 ; 258$ : 1373-1384.

7 Wannez S, Heine L, Thonnard M, et al. The repetition of behavioral assessments in diagnosis of disorders of consciousness. Ann Neurol $2017 ; 81$ : 883-889.

8 Sherer M, Yablon SA, Nakase-Richardson R, et al. Effect of Severity of Post-Traumatic Confusion and Its Constituent Symptoms on Outcome After Traumatic Brain Injury. Arch Phys Med Rehabil 2008 ; 89 : 42-47.

9 Laureys S, Pellas F, Van Eeckhout P, et al. The locked-in syndrome : what is it like to be conscious but paralyzed and voiceless? Prog Brain Res $2005 ; 150$ : 495-511.

10 Bruno M-A, Ledoux D, Vanhaudenhuyse A, et al. Prognosis of Patients with Altered State of Consciousness. In: Coma and Disorders of Consciousness.Springer London; 2012. pp. 11-23.

11 Schnakers C, Monti MM. Disorders of consciousness after severe brain injury. Curr Opin Neurol $2017 ; 30: 573-579$.

12 Demertzi A, Ledoux D, Bruno M-A, et al. Attitudes towards end-of-life issues in disorders of consciousness: a European survey. J Neurol 2011 ; 258 : 1058-1065. 
Seel RT, Sherer M, Whyte J, et al. Assessment scales for disorders of consciousness: Evidencebased recommendations for clinical practice and research. Arch Phys Med Rehabil 2010 ; 91 : 1795-1813.

Giacino JT, Kalmar K, Whyte J. The JFK Coma Recovery Scale-Revised: Measurement characteristics and diagnostic utility. Arch Phys Med Rehabil 2004 ; 85 : 2020-2029. Wannez S, Gosseries O, Azzolini D, et al. Prevalence of coma-recovery scale-revised signs of consciousness in patients in minimally conscious state. Neuropsychol Rehabil 2017 ; 2011 : 110.

Wannez S, Vanhaudenhuyse A, Laureys S, et al. Mirror efficiency in the assessment of visual pursuit in patients in minimally conscious state. Brain Inj $2017 ; 31: 1429-1435$.

Cheng L, Gosseries O, Ying L, et al. Assessment of localisation to auditory stimulation in postcomatose states : use the patient 's own name. 2013.

Stender J, Gosseries O, Bruno M-A, et al. Diagnostic precision of PET imaging and functional MRI in disorders of consciousness: A clinical validation study. Lancet $2014 ; 384$ : 514-522.

Chatelle C, Majerus S, Whyte J, et al. A sensitive scale to assess nociceptive pain in patients with disorders of consciousness. J Neurol Neurosurg Psychiatry $2012 ; 83$ : 1233-1237. Ommen HJ Van, Thibaut A, Vanhaudenhuyse A, et al. Resistance to eye opening in patients with disorders of consciousness. J Neurol 2018 ; $265: 1376-1380$. wakefulness syndrome ? J Neurol $2018 ; 265$ : 954-961.

22 Guldenmund P, Soddu A, Baquero K, et al. Structural brain injury in patients with disorders of consciousness: A voxel-based morphometry study. Brain Inj 2016 ; 30 : 343-352.

23 Velly L, Perlbarg V, Boulier T, et al. Use of brain diffusion tensor imaging for the prediction of long-term neurological outcomes in patients after cardiac arrest: a multicentre, international, prospective, observational, cohort study. Lancet Neurol 2018 ; 17 : 317-326.

24 Owen AM, Coleman MR, Boly M, et al. Detecting Awareness in the Vegetative State. Science 
(80- ) $2006 ; 313: 1402-1402$.

25 Monti MM, Vanhaudenhuyse A, Coleman MR, et al. Willful Modulation of Brain Activity in Disorders of Consciousness. N Engl J Med 2010 ; 362 : 579-589.

26 Cruse D, Chennu S, Chatelle C, et al. Bedside detection of awareness in the vegetative state: a cohort study. Lancet (London, England) $2011 ; 378: 2088-2094$.

27 Chaudhary U, Xia B, Silvoni S, et al. Brain-Computer Interface-Based Communication in the Completely Locked-In State. PLoS Biol 2017 ; 15 : 1-25.

Stoll J, Chatelle C, Carter O, et al. Pupil responses allow communication in locked-in syndrome patients. CURBIO; 23 : R647-R648.

Ruf CA, De Massari D, Wagner-Podmaniczky F, et al. Semantic conditioning of salivary pH for communication. Artif Intell Med $2013 ; 59$ : 91-98.

Charland-Verville V, Lesenfants D, Sela L, et al. Detection of response to command using voluntary control of breathing in disorders of consciousness. Front Hum Neurosci $2014 ; 8$ : 1020.

31 Boly M, Garrido MI, Gosseries O, et al. Preserved feedforward but impaired top-down processes in the vegetative state. Science $2011 ; 332: 858-862$.

Perrin F, Schnakers $C$, Schabus $M$, et al. Brain response to one's own name in vegetative state, minimally conscious state, and locked-in syndrome. Arch Neurol $2006 ; 63: 562-569$.

Demertzi A, Antonopoulos G, Heine L, et al. Intrinsic functional connectivity differentiates minimally conscious from unresponsive patients. Brain $2015 ; 138: 2619-2631$.

34 Chennu S, Annen J, Wannez S, et al. Brain networks predict metabolism, diagnosis and prognosis at the bedside in disorders of consciousness. Brain $2017 ; 140: 2120-2132$. Engemann D, Raimondo F, King JR, et al. Robust EEG-based cross-site and cross-protocol classification of states of consciousness. Brain 2018 ; In Press .

36 Casarotto S, Comanducci A, Rosanova M, et al. Stratification of unresponsive patients by an independently validated index of brain complexity. Ann Neurol $2016 ; 80: 718-729$. 
37 Gosseries O, Zasler ND, Laureys S. Recent advances in disorders of consciousness: Focus on the diagnosis. Brain Inj $2014 ; 28: 1141-1150$.

38 Gosseries O, Charland-Verville V, Thonnard M, et al. Amantadine, apomorphine and zolpidem in the treatment of disorders of consciousness. Curr Pharm Des $2014 ; 20: 4167-84$. Traumatic Brain Injury. Bad Aibling — both Ger Braintree Rehabil Hosp N Engl J Med 2012 ; $366: 819-26$.

40 Whyte J, Rajan R, Rosenbaum A, et al. Zolpidem and Restoration of Consciousness. Am J Phys Med Rehabil 2014 ; 93 : 101-113.

41 Yamamoto T, Katayama Y, Kobayashi K, et al. Deep brain stimulation for the treatment of vegetative state. Eur J Neurosci $2010 ; 32$ : 1145-1151.

42 Magrassi L, Maggioni G, Pistarini C, et al. Results of a prospective study (CATS) on the effects of thalamic stimulation in minimally conscious and vegetative state patients. J Neurosurg 2016 ; 125 : 972-981.

43 Thibaut A, Bruno M-A, Ledoux D, et al. tDCS in patients with disorders of consciousness Shamcontrolled randomized double-blind study. Neurology $2014 ; 82$ : 1112-1118.

44 Xia X, Bai Y, Zhou Y, et al. Effects of $10 \mathrm{~Hz}$ repetitive transcranial magnetic stimulation of the left dorsolateral prefrontal cortex in disorders of consciousness. Front Neurol $2017 ; 8: 1-8$. Corazzol M, Lio G, Lefevre A, et al. Restoring consciousness with vagus nerve stimulation. Curr. Biol. 2017; 27 : R994-R996. Monti MM, Schnakers C, Korb AS, et al. Non-Invasive Ultrasonic Thalamic Stimulation in Disorders of Consciousness after Severe Brain Injury: A First-in-Man Report. Brain Stimul. 2016; 9 : 940-941. 